\title{
Spontaneous fracture of the ulna complicating tuberculous osteomyelitis
}

\author{
D.J. Seddon, T. Thanabalasingham ${ }^{1}$ and J. Weinberg \\ Department of Medicine, Charing Cross Hospital, Fulham Palace Road, London W6 8RF and 'South Middlesex \\ Hospital, London, UK.
}

\begin{abstract}
Summary: A 63 year old Asian woman presented with tuberculous osteomyelitis of the right ulna and a large subcutaneous cold abscess. During the course of her treatment the midshaft of the ulna sustained a spontaneous transverse fracture. Aspects of her case are reviewed.
\end{abstract}

\section{Introduction}

Tuberculosis of the bones and joints is now uncommon in the United Kingdom. The majority of patients who are notified originate from the Indian subcontinent. In most cases, the vertebral column and intervertebral discs are involved.$^{1,2}$ However, infection may involve almost any bone in the body and may be present at more than one site in the skeleton at the same time ${ }^{3,4}$ Tuberculous osteomyelitis usually results from spread of infection from a primary site either in the lung or the bowel.

Pathological fracture of a bone damaged by tuberculosis is very rare. ${ }^{5,6} \mathrm{We}$ would like to report a case.

\section{Case report}

A 57 year old Asian woman was referred to this hospital with swelling of the right forearm. She had been born in India but had lived in the United Kingdom for 12 years. She had first noticed a localized swelling over the posterior aspect of her forearm 4 months previously. This had progressively enlarged but caused her no discomfort. There was no history of night sweats, fever, weight loss, or cough and her general health was good.

On examination, she was afebrile and had a tense mass involving the extensor muscle compartment of the right forearm. There was little inflammation of the skin and there was no bony tenderness. Physical examination of her chest and abdomen were unremarkable.

Investigations performed included radiographic examination of the right forearm, which showed soft tissue swelling, periosteal reaction and elevation and

Correspondence: D.J. Seddon, M.A., M.R.C.P.

Accepted: 12 May 1989 marked disorganization of the structure of the ulna bone. A chest radiograph, full blood count, urea, calcium and electrolytes and liver function tests were all normal. The ESR was $68 \mathrm{~mm}$ per hour.

Viscous yellow-white pus was aspirated from the abscess. No organisms were seen on light microscopy but culture revealed a growth of Mycobacterium tuberculosis. This was fully sensitive to rifampicin, isoniazid and pyrazinamide. When the results of the aspirate culture were available, she was commenced on rifampicin $10 \mathrm{mg}$ per $\mathrm{kg}$, isoniazid $200 \mathrm{mg}$ and pyrazinamide $1200 \mathrm{mg}$ daily, together with pyridoxine $10 \mathrm{mg}$.

Out-patient follow up showed that the cold abscess was failing to resolve and it was felt that the patient had not fully complied with treatment. She was therefore admitted for supervision of antibiotic therapy.

One week after admission, she suddenly developed pain and local bony tenderness over the site of the abscess and a radiograph revealed a transverse fracture of the mid-shaft of the ulna (Figure 1). There was no history of trauma. This was treated by drainage of the cold abscess and excision of necrotic bone with plaster cast immobilization and she has since made a full recovery.

\section{Discussion}

Pathological fracture complicating tuberculous osteomyelitis is rare and is not discussed in standard textbooks of pathology or orthopaedics. Even in large series of case ${ }^{2,4,7-10}$ it is not mentioned. In this patient we assume that the fracture occurred spontaneously as a result of the advanced state of destruction of the infected bone. The patient was in hospital under observation when the fracture occurred so that we can 


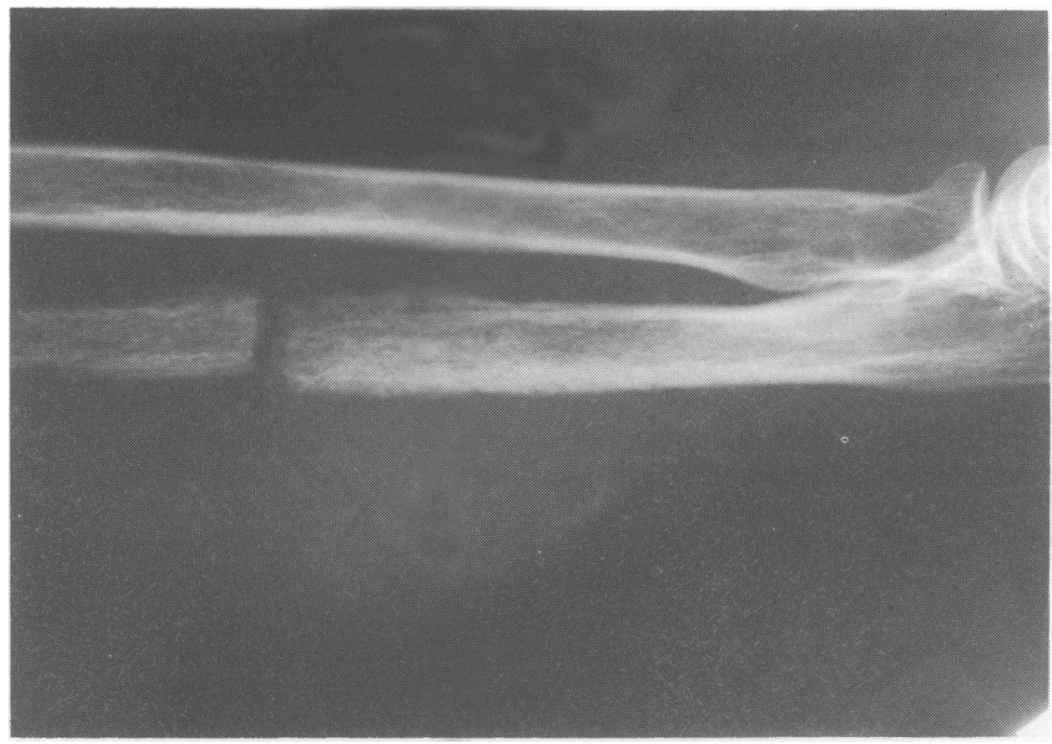

Figure 1 Radiograph of right forearm showing periosteal elevation, destruction of normal bone architecture and a transverse fracture of the mid-shaft of the ulna.

exclude significant trauma as a contributory factor. Figure 1 shows loss of normal bone architecture extending almost throughout the length of the ulna, and not just at the fracture site itself. In weight-bearing bones, spontaneous fracture might be expected to occur at an earlier stage in the progression of the disease.

\section{References}

1. Fancourt, G.J., Ebden, P., Garner, P., Cookson, J.B., Wales, J.M. \& Stoyle, T.F. Bone tuberculosis: results and experience in Leicestershire. Br J Dis Chest 1986, 80: 265-272.

2. Davies, P.D.O., Humphries, M.J., Byfield, S.P. et al. Bone and joint tuberculosis. J Bone Joint Surg (Br) 1984, 66B: 326-330.

3. Martini, M., Adjrad, A. \& Boudjemaa, A. Tuberculous osteomyelitis. A review of 125 cases. Int Orthop 1986, 10: 201-207.

4. Medical Research Council. National survey of tuberculosis notifications in England and Wales 1978-9. Br Med J 1980, 281: 895-898.

5. Jenyo, M.S. \& Komolafe, F. Tuberculous pathological fracture of the femur in a 15 year old boy. Pediatr Radiol 1986, 16: $260-261$.
Tuberculous osteomyelitis is frequently accomo panied by evidence of disease in the chest $\Phi$ abdomen. ${ }^{1}$ This patient had no obvious evidence of such infection at either of these sites though bariun studies were not performed. Though not unique, this unusual case demonstrates that pathological fracture may complicate tuberculous osteomyelitis.

6. Watts, R.A., Paice, E.W. \& White, A.G. Spontaneous fracture of the sternum and sternal tuberculosis. Thorax 1987, 42: 984-985.

7. Medical Research Council. National survey of tuberculosis notifications in England and Wales in 1983: characteristics of disease. Tubercle 1987, 68: 19-32.

8. Autzen, B. \& Elberg, J.J. Bone and joint tuberculosis in Denmark. Acta Orthop Scand 1988, 59: 50-52.

9. Nicholson, R.A. Twenty years of bone and joint tuberculosis in Bradford. J Bone Joint Surg (Br) 1974, 56B: $760-765$.

10. Newton, P., Sharp, J. \& Barnes, K.L. Bone and joint tuberculosis in Greater Manchester 1969-1979. Ann Rheum Dis 1982, 41: 1-6. 